\title{
Remesas familiares y sus efectos en la pobreza
}

Family remittances and their impact on poverty

Remessas familiares e seu impacto sobre a pobreza

DOI: http://dx.doi.org/10.23913/ricsh.v6i11.105

Ana Margarita Alvarado Juárez

Instituto de Investigaciones Sociológicas, Universidad Autónoma "Benito Juárez" de Oaxaca,

México

ana_alvarado38@hotmail.com

\section{Resumen}

En este trabajo se muestra el papel que juegan las remesas en la reducción de la pobreza en el municipio de San Lucas Quiaviní, Oaxaca. Para ello se consideraron las líneas de ingreso empleadas por el Consejo Nacional de Evaluación de la Política de Desarrollo Social (CONEVAL, 2010) en la medición multidimensional de la pobreza: la Línea de Bienestar (LB) y la Línea de Bienestar Mínimo (LBM). En términos metodológicos, se realizó una encuesta a los jefes de hogar de dicha comunidad para conocer la estructura del ingreso familiar. Los resultados reflejan que, considerando la línea de bienestar mínimo de $\$ 838.20$, la recepción de remesas reduce el porcentaje de hogares en pobreza alimentaria de 36.3 a $52 \%$; mientras que si se considera la línea de bienestar de \$1 555.10 las remesas reducen el porcentaje de 77.5 a $88.2 \%$. Palabras clave: migración, remesas, pobreza, Oaxaca.

\section{Abstract}

This paper shows the role played by remittances in the reduction of poverty in the municipality of San Lucas Quiavini, Oaxaca. Income lines used by the National Council for the Evaluation of Social Development Policy (CONEVAL by its name in Spanish, 2010) were considered the multidimensional poverty measurement: the Wellness Line (LB by its name in Spanish) and the Minimal Wellness Line (LBM by its name in Spanish). In methodological terms, a survey was 
conducted to the heads of the community home to learn the structure of household income. The results reflect that, considering the Minimal Wellness Line of $\$ 838.20$, remittance receipts reduces the percentage of households in poverty food from $36.3 \%$ to $52 \%$; conversely, considering the Wellness Line of $\$ 1555.10$ remittances reduce the percentage from $77.5 \%$ to $88.2 \%$.

Key words: migration, remittances, poverty, Oaxaca.

\section{Resumo}

Neste trabalho o papel das remessas na redução da pobreza no município de San Lucas Quiaviní, Oaxaca é mostrado. Linha Bem-estar (LB) e Linha Bem-estar mínimo (LBM): Para este rendimento linhas finais empregado pelo Conselho Nacional de Avaliação da Política de Desenvolvimento Social (CONEVAL, 2010) na medição multidimensional da pobreza que foram consideradas. Em termos de metodologia, foi realizada uma pesquisa para chefes de família da comunidade para conhecer a estrutura da renda familiar. Os resultados mostram que, considerando a linha mínimo de bem-estar de US $\$ 838,20$, recebendo remessas reduz a percentagem de famílias que vivem abaixo da breadline de 36,3 para 52\%; enquanto ele está considerando a linha de bem-estar $\$ 1555,10$ remessas reduzir o percentual de 77,5-88,2\%. Palavras-chave: migração, as remessas, a pobreza, Oaxaca.

Fecha recepción: Junio $2016 \quad$ Fecha aceptación: Diciembre 2016

\section{Introducción}

La migración de mexicanos hacia Estados Unidos es un fenómeno de larga historia y de importantes implicaciones en el ámbito económico, político y social. Dentro de la gama de manifestaciones de la movilidad de personas se encuentra el envío de dinero desde el extranjero hacia las comunidades de origen. En los últimos años, en nuestro país, el monto de las remesas ha sido comparado con indicadores macroeconómicos como el turismo y el petróleo.

Las remesas se han integrado a los patrones de ingreso y consumo de una parte importante de las familias mexicanas, por lo que cabe esperar que modifiquen las economías locales y regionales. 
En este contexto, el presente trabajo pretende aportar elementos empíricos que contribuyan a la discusión sobre el tema de las remesas y su relación con la reducción de la pobreza. Uno de los hallazgos de la investigación realizada es que las remesas logran cubrir las necesidades básicas de la población, en materia principalmente de compra de productos básicos para la alimentación y pago de servicios en la vivienda. Sin embargo, estos recursos representan una superación momentánea de la pobreza, puesto que son ingresos que no tienen una regularidad constante, lo que no permite efectos multiplicadores en la economía así como cambios estructurales.

Para demostrar lo anterior, se expone, en primer lugar, la metodología utilizada en la investigación, que consistió básicamente en la aplicación de un cuestionario dirigido a la unidad familiar, cuyo objetivo se orientó a detectar la composición del ingreso familiar. Con los resultados, se determinaron los efectos de las remesas sobre la incidencia, brecha y gravedad de la pobreza alimentaria, considerando las líneas de ingreso empleadas por el Consejo Nacional de Evaluación de la Política de Desarrollo Social (CONEVAL, 2010) para la medición multidimensional de la pobreza: la Línea de Bienestar (LB) y la Línea de Bienestar Mínimo (LBM). Posteriormente, se incluyen en este documento los resultados obtenidos de la encuesta antes mencionada que se aplicó en el municipio de San Lucas Quiaviní. También se describe el contexto en el que se vive la salida de personas hacia Estados Unidos. Enseguida, se presenta la discusión teórica del fenómeno analizado con los planteamientos de autores especialistas del tema. Finalmente, se ubica el apartado de conclusiones.

\section{La importancia del estudio de la migración y las remesas en México}

La migración de mexicanos hacia Estados Unidos es un fenómeno observado desde la segunda mitad del siglo XIX y principios del XX ante la confluencia de dos factores: el primero, relacionado con la expansión de la economía estadounidense basada en el desarrollo del ferrocarril, la agricultura y la minería que exigían mano de obra barata, abundante y poco calificada; el segundo, vinculado con las difíciles condiciones económicas y sociales que se vivían en México (Alanis, 2007).

A partir de ese momento, y a lo largo del siglo XX, adquiere diferentes particularidades, muchas de ellas relacionadas con las políticas migratorias implementadas por Estados Unidos e 
impulsadas por diferentes causas, entre las que se encuentran: los requerimientos de mano de obra en los sectores agrícola, industrial y de servicios, la diferencia salarial entre ambos países, la incapacidad de la economía mexicana para emplear su fuerza de trabajo, así como el apoyo de las redes sociales y familiares.

Este movimiento poblacional se ha incrementado a lo largo del tiempo. Así, de acuerdo con la oficina del censo de Estados Unidos, había 759000 mexicanos en 1970; 2.2 millones en 1980; 4.3 millones en 1990 y 9 millones de personas para el año 2000. En el año 2004, la población de origen mexicano con residencia en Estados Unidos se estimó en 26.6 millones de personas entre emigrantes nacidos en México (10.2 millones) y ciudadanos estadounidenses de ascendencia mexicana.

Es importante mencionar que los mexicanos representan la población hispana más grande que vive en Estados Unidos, esto es, alrededor del 64 \% de la población total de hispanos. En cuanto a las edades, seis de cada diez inmigrantes mexicanos en Estados Unidos tienen entre 15 y 44 años, mayoritariamente varones. El promedio de edad se ubica en 26 años. El mercado laboral en el que se emplean los mexicanos se orienta básicamente en trabajos de baja calificación y remuneración, de esta manera 1 de cada 4 mexicanos se emplea en actividades relacionadas con la preparación de alimentos, mantenimiento y limpieza de edificios; $21 \%$ se ocupa en el área de la producción y el transporte, y $20 \%$ en actividades de la construcción y reparación (López, 2015).

La decisión de migrar implica la realización de un proyecto individual o familiar, que se traduce en la adquisición de una vivienda, obtener un ingreso mejor remunerado, financiar la educación de los hijos, en términos generales, mejorar las condiciones materiales en las que vive el migrante y su familia. Este sueño impulsa el envío de remesas a las comunidades de origen. Enseguida se analiza la importancia de este tipo de ingresos.

Actualmente, el monto de los recursos que se reciben en México por concepto de remesas, es comparado con indicadores macroeconómicos nacionales como el turismo y el petróleo. De esta manera, en el año 2005 las remesas representaron la segunda fuente más importante de divisas, solamente superadas por el petróleo. En los últimos diez años, el flujo de remesas se ha caracterizado por importantes variaciones: en el 2007 registraron su máximo histórico de 26059 
millones de dólares, pero en 2008, 2009 y 2010 se redujeron, siendo la caída más importante en el año 2010 con un monto de 21304 millones de dólares. Para el año 2015, se transfirieron un total de 24790 millones de dólares (Banco de México, 2016).

Cabe resaltar que los envíos provenientes de Estados Unidos tienen como destino un espacio más o menos acotado del territorio nacional y constituyen un recurso económico fundamental para el sostenimiento familiar en las comunidades receptoras. Bajo esta perspectiva, las cifras nos indican que durante el año 2015, tres estados de la república mexicana recibieron, cada uno, más de dos mil millones de dólares; estos fueron: Michoacán (2 530 millones de dólares), Guanajuato (2 262 millones de dólares) y Jalisco (2 217 millones de dólares). Mientras que otros seis estados recibieron, cada uno, más de mil millones de dólares por remesas: Estado de México (1 560 millones de dólares), Puebla (1 370 millones de dólares), Oaxaca (1 288 millones de dólares), Guerrero (1 277 millones de dólares), Distrito Federal (1 089 millones de dólares) y Veracruz (1 085 millones de dólares). En conjunto estas nueve entidades captaron más de catorce mil millones de dólares, 59 \% del total de remesas. Es importante mencionar que la remesa promedio calculada para el año 2015 fue de 292 dólares (Banco de México, 2016).

En este análisis resulta pertinente considerar que el estado de Oaxaca se caracteriza por sus altos niveles de pobreza. El Consejo Nacional de Evaluación de la Política de Desarrollo Social ubicó a Oaxaca como la tercera entidad con mayor porcentaje de personas en situación de pobreza multidimensional, esto es $67.2 \%$ respecto del total de su población, superado por Chiapas con $78.4 \%$ y Guerrero con $67.4 \%$ (CONEVAL, 2012). Si se retoman las líneas de bienestar, es decir, el valor monetario de una canasta de alimentos, bienes y servicios básicos para los individuos, $36.8 \%$ de la población en Oaxaca se ubicó por debajo de esta línea y $68.6 \%$ por debajo de la línea de bienestar mínimo, que muestra únicamente el valor de la canasta alimentaria.

\section{La relación entre remesas y pobreza}

Sobre la relación entre remesas y pobreza existe literatura especializada que ha abordado este tema, llegando a conclusiones variadas, por ejemplo, Adams y Page (2005) analizaron, mediante una técnica cross-section, el efecto de las remesas en una muestra de 71 países en desarrollo. Los 
resultados muestran que un incremento del $10 \%$ de las remesas per cápita reduciría en $3.5 \%$ la proporción de personas que viven debajo de la línea de pobreza.

Por su parte, Fajnzylber y López (2007) encuentran que las remesas tienen un efecto positivo en la reducción de la pobreza, la equidad, el crecimiento y la inversión; esto se debe a los incrementos en el ingreso per cápita de los países receptores de remesas. Las especificaciones microeconómicas (basadas en hogares receptores) y de sección cruzada (basadas en países) sugieren conclusiones similares, explicando que por cada $1 \%$ de aumento en la proporción de remesas al PIB, el segmento de población viviendo en estado de pobreza se reduciría $0.4 \%$. Sin embargo, aclaran que el impacto de las remesas sobre la pobreza varía entre países en función de su nivel general de desarrollo.

En Guatemala, un estudio sobre los efectos de las remesas internas e internacionales en la pobreza, concluye que las remesas internas representaron $13.4 \%$ del ingreso total per cápita del hogar, mientras que las remesas internacionales significaron $20.8 \%$. Los dos tipos de remesas reducen la pobreza, esta afirmación tiene como base el análisis de la estructura de ingresos por deciles, en donde las remesas se concentraron en los hogares de más bajos ingresos. En el primer decil de los hogares con remesas internas, esta fuente constituyó $50 \%$ del total de ingresos en promedio y en los hogares con remesas internacionales éstas conformaron $60 \%$ del ingreso familiar (Adams, 2004).

Resultados contrapuestos a los obtenidos en el caso anterior son presentados por Gosh (2006), quien afirma que, aunque existe una minoría de receptores pobres, la mayor parte de los migrantes no provienen de hogares en pobreza. En este sentido, de acuerdo con este autor, de existir el vínculo entre remesas y reducción de pobreza, éste sería indirecto y se daría por spillover de las remesas recibidas por las familias de estos migrantes (Gosh, 2006).

Para el caso de México, un estudio realizado en el medio rural del sur-sureste, región con baja participación en la migración internacional, identificó que las remesas provenientes de Estados Unidos representaron $6.4 \%$ y un aumento del $10 \%$ impactaría en la incidencia, profundidad y severidad de la pobreza en $0 \%,-0.11 \%$ y $-0.11 \%$ respectivamente. En la región centrooccidente, considerada con altos niveles de migración internacional, las remesas constituyeron $15.9 \%$. El mismo aumento del $10 \%$ en este tipo de ingresos, provocaría una disminución en la 
incidencia, profundidad y severidad de la pobreza en $1.68 \%, 1.65 \%$ y $1.64 \%$ respectivamente (Taylor, Mora, Adams y López, 2005).

Por su parte, Székely y Rascón (2004) identificaron que la reducción de la pobreza alimentaria, entre los años 2000 y 2002, se debe al efecto del Programa Oportunidades (48 \%) y al aumento en las remuneraciones (44\%), mientras que el efecto de las remesas en la reducción de la pobreza extrema en esos años fue del $2 \%$, siendo el mismo efecto en la pobreza de capacidades y $12 \%$ para el caso de la pobreza de patrimonio.

López-Feldman (2011) agrupó comunidades rurales mexicanas en función de su experiencia migratoria y de un porcentaje de personas adultas migrantes. La conclusión es que cuanto mayor sea el porcentaje de adultos con experiencia migratoria, más difundida estará la información en cada región, lo que contribuye a la difusión de la migración y las remesas a través de los niveles de ingresos. De esta manera, la región sur-sureste de México es calificada por este autor con bajo nivel de historia migratoria y, por lo tanto, los efectos de las remesas sobre la pobreza son inferiores en comparación con la región de alto nivel de historia migratoria.

Esquivel y Huerta (2006) estudiaron la relación entre remesas y la condición de pobreza alimentaria y de capacidades. Determinaron que las remesas disminuyen la probabilidad de ubicarse en condición de pobreza alimentaria en $7.7 \%$ y una reducción del $6.3 \%$ para la pobreza de capacidades. Para el caso de los hogares rurales, la recepción de remesas disminuye en $10 \%$ la probabilidad de que un hogar se coloque en pobreza patrimonial, lo que representa una reducción del $15 \%$ en las tasas de pobreza de los hogares rurales.

$\mathrm{Al}$ analizar los estudios que abordan el impacto de las remesas sobre la pobreza en México, puede decirse que este tema no ha aportado los elementos de análisis suficientes para llevar a conclusiones contundentes y determinar los efectos de este tipo de recursos. En este sentido, el presente trabajo pretende aportar elementos empíricos que contribuyan a la discusión sobre el tema de las remesas y su relación con la reducción de la pobreza. 


\section{Materiales y métodos}

El primer paso para estimar el efecto de las remesas sobre la pobreza fue la elaboración de un cuestionario que recolectó datos sobre la unidad familiar: número de integrantes, ocupación, escolaridad, número y características de los migrantes, principales ingresos procedentes de remesas, jornales y salarios, transferencias del gobierno, comercio y autoconsumo.

Posteriormente, se realizó una encuesta a una muestra, probabilística y aleatoria, de jefes de hogar del municipio de San Lucas Quiaviní, abarcando $24 \%$ de los hogares del municipio, esto es, 102 hogares de un total de 425 registrados en el censo de 2010.

Para cuantificar el efecto de las remesas sobre la pobreza, se utilizaron los indicadores de Foster et al. (1984). Esta metodología permite hacer simulaciones y cumplir con los objetivos planteados.

$P(y ; z)=\frac{1}{n z^{\alpha}} \sum_{i=1}^{q} g_{i}^{\alpha}$

Donde: $P$ es la medida de pobreza, $y=\left(y_{i}, y_{2}, \ldots, y_{n}\right)$ es un vector de ingreso de los hogares en orden creciente, $z>0$ es la línea predeterminada de pobreza, $g_{i}=z-y_{i}$ es el déficit del ingreso del $i$ - ésimo hogar en relación a la línea de pobreza, $q=q(y ; z)$ es el número de hogares en pobreza con ingreso no mayor a $z, n$ es el número total de hogares y $\alpha$ toma valores de 0,1 y 2 para obtener la incidencia, brecha y gravedad de la pobreza.

Reardon y Taylor (1994); Taylor et al. (2005); Mora y López (2010) señalan que para descomponer $P(y ; z)$ por fuentes de ingreso, y sustituyendo $y$ por la suma de ingresos de cada fuente $i$ se tiene:

$P(y ; z)=\frac{1}{n z^{\alpha}} \sum_{i=1}^{q}\left(z-\sum_{i=1}^{k} y_{k}\right)^{\alpha}$

El impacto en el nivel de pobreza originado por un cambio porcentual en una fuente de ingresos, $e$, sobre la pobreza, $d P(y ; z) / d e$, está dado por: 
$\frac{d P(y, e ; z)}{d e}=\frac{1}{n z^{\alpha}}\left[\sum_{i=1}^{q_{0}}-\propto g_{i}(e)-\sum_{q-} g_{i}(e)^{\propto}+\sum_{q+} g_{i}(e)^{\propto}\right]$

Donde:

$q-(q+)$ es el número de hogares que dejan la pobreza como resultado de un cambio en la fuente de ingresos.

Para determinar los efectos de las remesas sobre la pobreza se utilizaron los indicadores de: incidencia, brecha y gravedad de la pobreza alimentaria; además, se consideraron las líneas de ingreso calculadas por el Consejo Nacional de Evaluación de la Política de Desarrollo Social (CONEVAL, 2010) para la medición multidimensional de la pobreza: la Línea de Bienestar (LB) y la Línea de Bienestar Mínimo (LBM). El valor promedio en 2013 de las LB y LBM fue de $\$ 1555.1$ y $\$ 838.2$, respectivamente. Dichas líneas de ingreso son asumidas por $z$, de acuerdo a la metodología.

La incidencia es el porcentaje de hogares, con respecto al total de hogares, que se encuentran por debajo de la línea de pobreza; la brecha de la pobreza es la distancia promedio que separa a la población de la línea de pobreza, y la gravedad o severidad de la pobreza asume valores entre 0 y 1 , donde 0 muestra que la pobreza no es severa y 1 cuando la pobreza es sumamente severa.

La LB hace posible identificar a la población que no cuenta con los recursos suficientes para adquirir los bienes y servicios que requiere para satisfacer sus necesidades alimentarias y no alimentarias, mientras que la LBM permite identificar a la población que, aun haciendo uso del total de su ingreso en la compra de alimentos, no puede adquirir lo indispensable para tener una nutrición adecuada (CONEVAL, 2010).

Se realizaron dos escenarios, primero considerando el ingreso total en el hogar y el segundo sin las remesas internacionales. Los escenarios se realizaron usando el comando poverty en el programa Data Analysis and Statistical Software (STATA, 2009).

A continuación se presentan los resultados del procesamiento de los datos obtenidos en el trabajo de campo, así como información del contexto en el que se vive la migración de la comunidad en estudio. 


\section{Resultados}

El municipio de San Lucas Quiaviní pertenece al distrito de Tlacolula, uno de los 7 que conforman la Región de Valles Centrales de Oaxaca. Se localiza en las coordenadas $16^{\circ} 54^{\prime}$ de latitud norte y $96^{\circ} 28^{\prime}$ de longitud oeste, a una latitud de $1730 \mathrm{msnm}$. Colinda al norte con el municipio de Tlacolula de Matamoros; al sur con Santiago Matatlán; al oeste con Bartolomé Quialana; al este con los municipios de Santiago Matatlán y Tlacolula de Matamoros. La distancia entre esta comunidad y la ciudad de Oaxaca es de 50 kilómetros.

Cuenta con una extensión aproximada de $58.69 \mathrm{~km}^{2}$, lo que representa $0.061 \%$ del total del estado, su superficie está conformada en su mayor parte por planicies, aunque también presenta lomeríos, además de estar rodeado por diversos cerros. Sus principales elevaciones son el cerro Quiaviní y el cerro San Felipe. Sus recursos hidrográficos son escasos, por lo que el arroyo más importante es el río Principal, sólo mantiene su afluente en época de lluvias. Su clima es templado con lluvias en verano y frío en invierno, similar al clima de la capital oaxaqueña.

En lo que se refiere a las actividades económicas, la mayor parte de la población económicamente activa se dedica a las actividades agrícolas. La agricultura que se practica es de temporal y se destina, fundamentalmente, al autoconsumo. Los cultivos principales son el maíz y el frijol. En menor medida existe la producción por sistema de riego cuyo producto es la alfalfa. La siembra de agave se ha descontinuado en virtud de los bajos precios del mercado. Según los datos del Censo de 2010, la población económicamente activa fue de 174 personas y la población económicamente no activa ascendió a 601, entre las que se encuentran las personas pensionadas o jubiladas, estudiantes o dedicadas a los quehaceres del hogar (INEGI, 2010).

En este municipio la mayoría de los habitantes hablan lengua indígena, específicamente, zapoteco. En este sentido, el censo del año 2010 registró 1591 personas hablantes de zapoteco, de las cuales 643 fueron hombres y 948 mujeres; 1277 hablan español, 356 no hablan español y 46 no hablan lengua indígena (INEGI, 2010).

En cuanto a la dinámica demográfica se observa que la población total del municipio ha disminuido en los últimos años, por ejemplo, en 1980 el número de habitantes era 2 127; para 
1990 se registraron 2 156; en el año 2000 la cifra fue de 1941 y para el 2010 se contaban con 1 745 habitantes. Estas cifras son reflejo tanto del crecimiento natural de la población, es decir, nacimientos y defunciones, como del crecimiento social: emigraciones e inmigraciones. En este caso, el descenso poblacional registrado en las últimas décadas es resultado, fundamentalmente, de la emigración y las bajas tasas de natalidad.

La pirámide poblacional para el año 2010 nos muestra que la mayoría de las personas se encuentran en edades menores de 20 años. Se observa que en edades productivas existe una disminución, principalmente de hombres, en edades entre los 20 y 44 años, quienes emigran en busca de mejores oportunidades de empleo. La barra más larga de la pirámide es para las edades de 15 a 19 años.

Al hacer un recorrido por las calles de esta comunidad es común observar, en mayor medida, a mujeres; este fenómeno también se encuentra registrado en las estadísticas al contar con un índice de masculinidad de 70, lo que significa que existen 70 hombres por cada 100 mujeres.

En este sentido, en el año 2015 San Lucas Quiaviní presentó un grado de marginación muy alto que se refleja en las condiciones precarias en las viviendas, con hacinamiento y carencia de servicios, asimismo se observan altos índices de población analfabeta o con estudios limitados que impiden integrarse a un mercado de trabajo competitivo; de igual forma, la población ocupada obtiene ingresos bajos que no le permiten obtener los requerimientos indispensables para la vida como una alimentación adecuada en cantidad y calidad (CONAPO, 2016).

Las cifras del CONEVAL para este municipio indican que $75.5 \%$ de la población se encuentra en situación de vulnerabilidad de ingreso, de éstas $41.2 \%$ vive en pobreza moderada y $34.3 \%$ en pobreza extrema. Algunos indicadores elaborados por este organismo se reproducen a continuación:

+ El grado promedio de escolaridad de la población de 15 años o más en el municipio fue, en 2010, de 3.8 frente al grado promedio de escolaridad de 6.9 en la entidad.

$\phi$ En 2010, 1238 individuos (75.5\% del total de la población) se encontraban en pobreza, de los cuales $676(41.2 \%)$ presentaban pobreza moderada y 562 (34.3\%) pobreza extrema. 
\$ En 2010, la condición de rezago educativo afectó a $60.8 \%$ de la población, lo que significa que 997 individuos presentaron esta carencia social.

$\phi$ En el mismo año, el porcentaje de personas sin acceso a servicios de salud fue de $47.8 \%$, equivalente a 783 personas.

\$ La carencia por acceso a la seguridad social afectó a 93.8 \% de la población, es decir, 1 539 personas se encontraban en esta condición.

\$ El porcentaje de individuos que reportó habitar en viviendas con mala calidad de materiales y espacio insuficiente fue de $45.3 \%$ (743 personas).

\$ El porcentaje de personas que reportó habitar en viviendas sin disponibilidad de servicios básicos fue de $71.7 \%$, lo que representa que las condiciones de vivienda no son las adecuadas para 1176 personas.

\$ La incidencia de la carencia por acceso a la alimentación fue de $16.5 \%$, es decir, una población de 271 personas (CONEVAL, 2014).

Las cifras anteriores nos ofrecen un panorama de la situación que se vive en el municipio de San Lucas Quiaviní, pues al visitar esta comunidad se observan sus carencias en términos de situación de vida. En este contexto, algunos pobladores han visto en la emigración una opción para mejorar sus condiciones económicas.

En lo que se refiere a estadísticas en materia migratoria, para el año 2010 el CONAPO estimó que $23.53 \%$ de los hogares tiene familiares en Estados Unidos, $2.59 \%$ cuenta con migrantes circulares, estos son los que viajan por temporadas hacia diferentes destinos y regresan a la comunidad; $8 \%$ de las familias tiene experiencia de migración de retorno y $48.7 \%$ de los hogares recibe remesas (CONAPO, 2012).

Se trata de una emigración principalmente de hombres jóvenes que tienen como destino el estado de California en los Estados Unidos. Las ocupaciones principales de los migrantes se orientan hacia el sector servicios, principalmente en restaurantes. Como consecuencia de las dificultades para cruzar la frontera y los elevados costos de traslado, los migrantes se van por periodos entre 2 y 4 años. Esto ha provocado que las mujeres se responsabilicen de las actividades productivas locales, como es el caso del cultivo de la tierra. 
Sobre la importancia de las remesas para esta comunidad, los resultados del trabajo de campo permitieron identificar que este tipo de recursos significaron $18.6 \%$ con respecto al ingreso total de los hogares. Considerando el total de remesas y su distribución en los deciles de hogares, se encontró que $38.9 \%$ del total de las remesas se encontraba concentrado en el decil de más altos ingresos, mientras que solo $0.8 \%$ se ubicó en el primer decil. Esto significa que no son los hogares de mayor pobreza quienes reciben remesas.

El ingreso promedio per cápita mensual del hogar fue de $\$ 1124.10$, de los cuales $\$ 228.60$ correspondieron a remesas internacionales. El efecto de fuente de ingresos se muestra en el cuadro I. Resalta que las remesas internacionales reducen el porcentaje de hogares en pobreza alimentaria de 36.3 a $52 \%$.

Cuadro I. Indicadores de pobreza con y sin remesas, San Lucas Quiaviní, 2013.

\begin{tabular}{|l|c|c|c|c|c|c|}
\hline \multirow{2}{*}{ Línea de ingreso } & \multicolumn{2}{|c|}{ Con remesas internacionales } & \multicolumn{2}{c|}{ Sin remesas internacionales } \\
\cline { 2 - 7 } & Incidencia & Brecha & Severidad & Incidencia & Brecha & Severidad \\
\hline $\begin{array}{l}\text { Línea de bienestar } \\
\text { Mínimo }\end{array}$ & 36.3 & 12.5 & 6.9 & 52.0 & 21.7 & 13.2 \\
\hline Línea de Bienestar & 77.5 & 34.9 & 19.7 & 88.2 & 45.3 & 28.7 \\
\hline
\end{tabular}

Fuente: elaboración propia.

\section{Discusión}

Para la perspectiva crítica, la migración de mexicanos a Estados Unidos representa un fenómeno eminentemente laboral y como tal, los ingresos obtenidos por los migrantes significan un fondo salarial que, como cualquier otro, tiende a usarse preferentemente en el sustento material de la familia. Por lo anterior, las remesas "no son consideradas ni como una forma de ahorro ni como una fuente para la inversión productiva, sino como un fondo salarial, que como tal se destina principalmente al consumo y la reproducción material del hogar" (Canales y Montiel, 2004, p.149).

Desde este panorama, al analizar la información recolectada en el trabajo de campo llevado a cabo en el municipio de San Lucas Quiaviní, se detecta que las remesas contribuyen a reducir la pobreza de las familias en esta comunidad, sin embargo, es importante considerar que estos 
recursos no están garantizados a lo largo del tiempo, al tratarse de ingresos temporales dependientes del empleo que tenga el migrante en Estados Unidos. Por lo tanto, este tipo de recursos no puede revertir la pobreza a largo plazo.

De acuerdo con Canales (2007), las remesas deben ser consideradas como un salario y como tal, tienen el mismo significado e impacto que cualquier otra categoría de remuneración al trabajo, enfocado, principalmente en la reproducción material de las familias. En este sentido, las remesas tienen un limitado impacto en el impulso al desarrollo y en la reducción de la pobreza, siendo en los estratos pobres con muchas carencias donde las remesas contribuyen a paliar la pobreza, pero en ningún caso a revertirla.

En San Lucas Quiaviní, las remesas significaron 18.6\% con respecto al ingreso familiar, esto se traduce en mejores condiciones de vida para la familia al destinarse a gastos de alimentación, vestido, salud, educación y vivienda. Es importante retomar el planteamiento de Corona (2014), quien detectó que las remesas se emplean dependiendo del proyecto migratorio, esto es, de los objetivos que tenga la familia y el migrante: los que emigran para obtener un salario, y el gasto se orienta en las necesidades básicas como la alimentación, posteriormente, lo invierten en educación, salud, la vivienda o adquisición de activos físicos. En el caso de San Lucas Quiaviní, los emigrantes, en su mayoría, salen en busca de un empleo bien remunerado para satisfacer sus necesidades básicas.

Corona (2014) coincide con Canales (2007) en que las remesas pueden verse como parte de un salario, en este caso un salario internacional, y concluye que las remesas tienen un efecto positivo para el desarrollo y bienestar de las familias, tomando a la migración como una estrategia seguida por los hogares para superar la pobreza en que viven. Este punto es importante si consideramos que para este municipio $75.5 \%$ de la población se encuentra en situación de vulnerabilidad de ingreso, donde las fuentes de empleo son reducidas y, por lo tanto, las personas ven en la migración una alternativa para superar la pobreza. Cabe recalcar que son los hombres jóvenes en edad productiva los que emigran para buscar un empleo en Estados Unidos.

Por otra parte, no es posible hablar de desarrollo económico impulsado por remesas en la comunidad que nos ocupa, debido a que estos ingresos no son invertidos en proyectos que generen ganancias o en empresas que impulsen efectos multiplicadores, esto es porque los 
recursos no son suficientes para el ahorro y la inversión. Además, el promedio de escolaridad de la población es de 3.8 grados, lo que implica una preparación insuficiente para impulsar las habilidades empresariales, aunado a esto los migrantes retornados no obtienen la capacitación necesaria, en el país de destino, al desempeñarse en puestos de baja calificación como meseros o ayudantes de cocina.

Esta situación que se observa en San Lucas Quiaviní, se puede analizar a la luz de los planteamientos de García, Peláez y Fuentes (2015), quienes encuentran que la tradición migratoria de una región contribuye a que una mayor proporción de remesas se destine a la inversión en negocios. Esta relación se ve favorecida por la edad de los emigrantes y de los jefes de hogares receptores, así como el mayor grado de desarrollo de las instituciones relacionadas con el proceso migratorio. Los autores encuentran que los hogares con mayor tradición migratoria dedican una mayor proporción de su presupuesto a negocios. Esto significa que la región tradicional de migración tendrá mayor propensión a invertir en negocios en comparación con otras regiones del país. Aunado a esto, las asociaciones de migrantes juegan un papel importante porque facilitan información sobre programas y proyectos de inversión, además de participar como enlace con las comunidades de origen. San Lucas Quiaviní no cuenta con las características anteriores al formar parte de una región de migración emergente, no hay inversión en negocios y no existen asociaciones de migrantes que impulsen proyectos productivos.

A lo anterior se le agrega la ausencia de fuentes públicas de financiamiento, que se traduce en el abandono por parte del Estado de políticas de bienestar, subsidios, créditos y, en general, apoyo a la pequeña empresa. También es notable la falta de financiamiento privado a través de bancos, instituciones financieras y empresas que participen en el financiamiento de proyectos productivos.

\section{Conclusiones}

Los recursos que se reciben en México por concepto de remesas es de tal magnitud que se han comparado con indicadores macroeconómicos como el petróleo y el turismo. Sin embargo, este tipo de recursos se reparte en un centenar de hogares pulverizando los grandes montos y convirtiéndose en pequeñas cantidades para solventar gastos de alimentación, educación y ahorros para invertir en construcción de vivienda. 
El debate sobre el impacto de las remesas se ubica entre quienes afirman que estos ingresos reducen la pobreza y la desigualdad del ingreso, estabilizan la economía familiar y contribuyen al desarrollo local de las comunidades y autores que sostienen que las familias se vuelven dependientes de las remesas y provocan el atraso económico. Además está la visión que considera a las remesas como un salario que se utiliza como tal. Los estudios de caso abonan hacia uno y otro argumento sin que hasta el momento se haya llegado a una conclusión definitiva.

En este contexto, la situación de pobreza que enfrentan los habitantes de San Lucas Quiaviní se ve reflejada en diferentes indicadores, tales como: ingresos económicos bajos, falta de acceso a oportunidades de empleo, carencia de servicios básicos en las viviendas, hacinamiento y analfabetismo. Aunado a lo anterior, la agricultura, que ha sido una actividad principal, enfrenta los problemas de tierras de mala calidad para la producción, ausencia de capacitación para el manejo de cultivos y escasez de agua.

Ante este panorama, los pobladores han optado por salir de su comunidad para lograr mejorar sus condiciones de vida mediante la búsqueda de empleos mejor remunerados. Así, la migración en esta comunidad es una estrategia que han seguido las familias durante cuatro décadas, enviando principalmente a los hombres jóvenes en edad productiva.

Los resultados de esta investigación identifican que el ingreso promedio per cápita mensual de los hogares en San Lucas Quiaviní fue de \$1 124.10, de los cuales \$228.60 correspondieron a remesas internacionales, con este tipo de ingresos los hogares reducen su porcentaje de pobreza alimentaria de 36.3 a $52 \%$, mientras que si se considera la línea de bienestar de \$1 555.10 las remesas reducen el porcentaje de 77.5 a $88.2 \%$. Es importante resaltar que para esta comunidad $38.9 \%$ del total de las remesas se encontraba concentrado en el decil de más altos ingresos, mientras que sólo $0.8 \%$ se ubicó en el primer decil, esto es, que no son los hogares de mayor pobreza los que reciben remesas.

Sin embargo, las remesas se convierten en un ingreso que les permite a las familias satisfacer sus necesidades básicas en materia, principalmente, de alimentación. Sin embargo, la superación de la pobreza mediante el envío de este tipo de recursos va a depender de los montos, frecuencia y los usos que se registren. Por todo lo anterior se debe considerar el contexto en el que se han 
realizado los estudios sobre el impacto de las remesas para determinar que el desarrollo local de las comunidades a partir de las remesas depende del entorno económico, social y político en el que se reciben, de la antigüedad de los flujos migratorios, de las oportunidades regionales de inversión y del capital humano que se traduce en las habilidades de los migrantes para invertir en proyectos productivos que se mantengan a lo largo del tiempo.

\section{Bibliografía}

Adams, R., y J. Page (2005) Do International Migration and Remittances Reduce Poverty in Developing Countries?, World Development, 33 (10), 1645-1669.

Adams, R. (2004) Remittances and poverty in Guatemala (Documento de Trabajo núm. 3418). World Bank Policy Research.

Alanís, F. (2007) Que se queden allá. El gobierno de México y la repatriación de mexicanos de Estados Unidos (19341940). México: El Colegio de la Frontera Norte-El Colegio de San Luis. Banco de México (2016). Remesas familiares. Recuperado de http://www.banxico.org.mx/.

Canales, A I; (2007). Remesas y pobreza en México. Una relación por explorar. Trayectorias, IX (25), 7-17. Recuperado de http://www.redalyc.org/articulo.oa?id=60715120003.

Canales, A. y Montiel I. (2004) Remesas e inversión productiva en comunidades de alta migración a Estados Unidos. El caso de Teocaltiche, Jalisco. Migraciones Internacionales, 2 (3), 142-172.

CONAPO (2016) Índice de marginación por entidad federativa y municipio, 2015. Recuperado de: http://www.gob.mx/conapo/documentos/

CONAPO (2012) Índices de intensidad migratoria México-Estados Unidos 2010. Recuperado de: http://www.conapo.gob.mx/

CONEVAL (2014) Medición de la pobreza. Resultados a nivel municipal. Recuperado de: http://www.coneval.gob.mx/.

CONEVAL (2012) Resultados de pobreza en México 2012 a nivel nacional y por entidades federativas. Recuperado de: http://www.coneval.gob.mx/.

CONEVAL (2010) Metodología para la medición multidimensional de la pobreza en México, México: CONEVAL. 
Corona, M. Á. (2014). Las remesas y el bienestar en las familias de migrantes. Perfiles Latinoamericanos, 43, 185-207.

Recuperado de http://www.redalyc.org/articulo.oa?id=11529850008.

Data Analysis and Statistical Software (STATA) (2009) Stata user's guide release 11. College Station, Texas: Stata Press Publications.

Esquivel, G. y Huerta-Pineda, A. (2006) Remittances and Poverty in México: A propensity Score Matching Approach, Inter-American Development Bank.

Fajnzylber, P. y López, H. (2007) Remittances and Development Lessons from Latin America. Banco Mundial.

Foster, J.; Greer J. and Thorbecke E. (1984) A class of decomposable poverty measures. Econometrica, 52, 761-766.

García J., Peláez O. y Fuentes N. A. (2015). La tradición migratoria como factor explicativo del uso de remesas en la financiación de negocios en Guanajuato. Migraciones internacionales, 8(2), 165-194.

Recuperado de http://www.scielo.org.mx/scielo.php?script=sci_arttext\&pid=S1665$89062015000200006 \& \operatorname{lng}=\mathrm{es} \& \operatorname{lng}=\mathrm{es}$.

Gosh, B. (2006), Migrants, Remittances and Development: Myths, Rhetoric and Realities, Organización Internacional de las Migraciones (OIM) y The Hague Process on Refugees and Migration, Ginebra

INEGI (2010) XIII Censo general de población y vivienda 2010. México: Instituto Nacional de Estadística, Geografía e Informática.

López, G. (2015): Hispanics of Mexican origin in the United States, 2013. Recuperado de http://www.pewhispanic.org/2015/09/15/hispanics-of-mexican-origin-in-the-united-states2013/

López-Feldman, A. (2011) Migration history, remittances and poverty in rural México. Economics Bulletin, 31, 1256-1264.

Mora, J. y López A. (2010) Efecto potencial de las remesas en la pobreza y la desigualdad, Panorama Económico, 6, 63-82.

Reardon, T. and Taylor E.(1994) Agroclimatic shock, income inequality and poverty: evidence from Burkina Faso. Staff Paper No. 94-27. Departament of Agricultural Economics, Michigan State University. 29p. 
Rendón, G., y V. González R. (1999). Cálculo simplificado de tamaños de muestra y valores tabulados. Comunicaciones en socioeconomía, estadística e informática, 3 (2), 4-77.

Székely, M. y Rascón, E. (2004) México 2000-2002: Reducción de la pobreza con estabilidad y expansión de programas sociales. Secretaría de Desarrollo Social. Serie: Documentos de Investigación.

Taylor, E., Mora J.; Adams R. and López A. (2005) Remittances, inequality and poverty: evidence from rural Mexico. Working paper 05-003. 